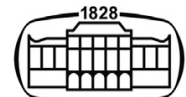

AKADÉMIAI KIADÓ

Acta Chromatographica

34 (2022) 1, 100-113

DOI:

10.1556/1326.2020.00825

(c) 2020 The Author(s)

ORIGINAL RESEARCH PAPER

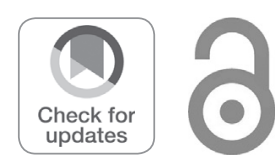

${ }^{*}$ Corresponding author. Department of Pharmaceutical Sciences, College of Pharmacy, Western University of Health Sciences, Pomona, CA 91766 , USA. Tel.: +1 909469 5220; fax: +1 9094695600.

E-mail: yhuang@westernu.edu

** Corresponding author. Department of Pharmaceutical Sciences, College of Pharmacy, Marshall B. Ketchum University, Fullerton, CA 92831, USA. Tel.: +1 714872 5719; fax: +1 714 8725706.

E-mail: zwang@ketchum.edu

\section{Quality evaluation of commercial products of Ganoderma lucidum made from its fruiting body and spore}

\author{
STEVEN YEUNG ${ }^{1}$, QUANLAN CHEN ${ }^{2}$, YONGBANG YU ${ }^{2}$, \\ BINGSEN ZHOU ${ }^{2}$, WEI WU ${ }^{2}, \mathrm{XIA} \mathrm{LI}^{2}$, YING HUANG ${ }^{1 *}$ (1) and \\ ZHIJUN WANG ${ }^{1,3 * *}$ (1) \\ ${ }^{1}$ Department of Pharmaceutical Sciences, College of Pharmacy, Western University of Health \\ Sciences, Pomona, CA 91766, USA \\ ${ }^{2}$ Beijing Tong Ren Tang Chinese Medicine Co., Ltd, Hong Kong, China \\ ${ }^{3}$ Department of Pharmaceutical Sciences, College of Pharmacy, Marshall B. Ketchum University, \\ Fullerton, CA 92831, USA
}

Received: July 28, 2020 • Accepted: December 25, 2020

Published online: February 26, 2021

\begin{abstract}
Ganoderma lucidum (GL), also known as Reishi or Lingzhi, is a medicinal mushroom widely used in traditional and folk medicines. The extracts made from the fruiting body and spore of naturally grown GL are the most frequently used in commercial products. More than 400 compounds have been identified in GL with the triterpenoids considered to be the major active components. Large variations in the chemical components were reported in previous studies and there is no comprehensive study of the content of multiple major triterpenoids in the GL product. In addition, there is no report in the comparison of chemical profiles in different parts of GL (i.e., fruiting body and spore). Determining the chemical composition and comparing the differences between fruiting body and spore are essential for the identity, efficacy and safety of various GL products.

In this study, 13 compounds (ganoderenic Acid C, ganoderic Acid C2, ganoderic Acid G, ganoderic Acid B, ganoderenic Acid B, ganoderic Acid A, ganoderic Acid H, ganoderenic Acid D, ganoderic Acid D, ganoderic Acid F, ganoderic Acid DM, ganoderol A, and ergosterol) were selected as the chemical markers. The purpose of this study is to develop an HPLC-DAD fingerprint method for quantification of these active components in GL (spore and fruiting body) and test the feasibility of using the HPLCDAD fingerprint for quality control or identity determination of GL products.

The results showed that this method could determine the levels of the major components accurately and precisely. Among the 13 components, 11 ganoderma acids were identified to be proper chemical markers for quality control of GL products, while ganoderal A was in a very low amount and ergosterol was not a specific marker in GL. The extracts of fruiting body contained more chemical compounds than those of spore, indicating that these 11 compounds could be a better chemical marker for the fruiting body than the spore. The HPLC chemical fingerprint analysis showed higher variability in the quality of GL harvest in different years, while lesser variation in batches harvested in the same year.

In conclusion, an HPLC assay detecting 11 major active components and a fingerprinting method was successfully established and validated to be feasible for quality control of most commercial GL products.
\end{abstract}

\section{KEYWORDS}

Ganoderma lucidum, HPLC fingerprint, quality control, chemical quantification, fruiting body, spore

\section{BACKGROUND AND AIM}

When ganoderma, a genus of polypore fungi, was established in 1881, included only one species, Ganoderma lucidum (Curtis) Pleurotus karst (GL). At present, more than 80 species 
have been identified all over the world, such as Ganoderma lingzhi, Ganoderma flexipes, and G. lucidum [1,2]. In China, it is also known as Reishi or Lingzhi, which has been used with a long history in traditional and folk medicines as a medicinal mushroom for promoting health, immune modulation, and longevity [3-6]. Recent studies revealed that GL possesses many pharmacological functions such as antimicrobial infection, anti-inflammation, anti-oxidation, antidiabetes and anti-cancer activities [7-9]. Various commercial products worldwide in different formulations either as medicine or dietary supplement (powder, capsule, pills, tea, decoction, etc.) are produced from different parts of the mushroom mainly including the fruiting body (matured from mycelium) or the spores. The fruiting body is the mushroom which makes spore, the reproductive unit.

The quality control of pharmaceutical products is essential for their identity, efficacy, and safety. Quantification of multiple chemical components in the herbal extract is the common quality control method for herbal medicine. At present, the high-performance liquid chromatography with UV detection (HPLC-UV) is the most frequently accepted method for the simultaneous determination of multiple constituents in G. lucidum and its related products [10-12]. Although mass spectrometry is able to provide better specificity and sensitivity, it has the limitations of interlaboratory heterogeneities and variability [13, 14]. The high-performance thin-layer chromatography was also reported to screen GL based on the peak profiles of polysaccharide hydrolyzates, but it is less sensitive and specific for chemical compounds [15-17].

More than 400 compounds have been identified in GL, among which triterpenoids are the major active components [18]. As a herb medicine, the content of each compound is highly affected by many factors, such as cultivation, harvest time, post-harvest processing, and storage conditions [19].

Although both the fruiting body and spore have been used for medicines, their difference in chemical composition is not well studied yet. This study is aimed to determine the chemical differences between the fruiting body and spore of GL using HPLC-DAD based HPLC fingerprint technology. The harvest time can be a factor leading to variation in their quality. Even from the same source, the quality can vary year by year depending on the weather conditions. However, there is little information about the inter-year variation of the GL raw materials collected in different years.

The purpose of this study is to develop an HPLC-DAD method for quantification of multiple main active components in GL (spore and fruiting body) and compare their chemical compositions. Besides, the HPLC-DAD fingerprint for quality control or identity determination of GL product was constructed and applied to determine the chemicals profiles of GL harvested in different years to determine the inter-batch variability. In this study, multiple batches of broken spore (GLS) and fruiting body (GLF) of GL collected in various years were tested in terms of their chemical components and their fingerprints were also established. A commercial product named GLSF, a new GL-derived product containing GLS and GLF mixed with a ratio of GLS:GLF $=4: 1(\mathrm{w} / \mathrm{w})$ based on principles of Traditional Chinese Medicine, was also included for quality evaluation.

\section{MATERIALS AND METHODS}

\section{Materials}

The standard extracts of GLS and GLF were obtained from the National Institute of Food and Drug Control (Beijing, China), which is used as the standard products for constructing the standard chromatogram for quality control.

The commercial products were provided by Ton Ren Tang (hereinafter referred to as TRT products), including nine batches of GLS collected in years of 2015 (batch 1 and 2), 2016 (batch 3, 4, and 5), 2017 (batch 6 and 7), and 2018 (batch 8,9 ), respectively, and 14 batches of GLF collected in years of 2013 (batch 1), 2014 (batch 2), 2015 (batch 3, 4, and 5), 2016 (batches 6-11), 2017 (batch 12 and 13), and 2018 (batch 14), respectively.

In addition, 5 batches of GLSF (GLS was mixed with GLF with the ratio of $4: 1, w / w$, batch $1,2,3,4,5)$ were also analyzed.

Based on the literature search the main active components including ganoderenic Acid C (GNAC), ganoderic Acid C2 (GAC2), ganoderic Acid G (GAG), ganoderic Acid B (GAB), ganoderenic Acid B (GNAB), ganoderic Acid A (GAA), ganoderic Acid H (GAH), ganoderenic Acid D (GNAD), ganoderic Acid D (GAD), ganoderic Acid F (GAF), ganoderic Acid DM (GADM), ganoderol A (GLA), and ergosterol (EGL) were selected as the quantification markers (Fig. 1) and purchased from Chinese pure standards market with purity higher than $90 \%$ based on their corresponding HPLC chromatograms.

HPLC grade acetonitrile, methanol, and water were purchased from Fisher Scientific (Pittsburgh, PA, USA). Chloroform for extraction of herbs was purchased from $\mathrm{BDH}$ Chemicals (VWR International, Radnor, PA).

\section{Methods}

\section{HPLC assay development and validation}

Standards preparation. The stock solutions of GNAC, GAC2, GAG, GAB, GNAB, GAA, GAH, GNAD, GAD, GAF, GADM, GLA, and EGL were prepared by dissolving appropriate amounts of each individual standards in methanol to generate a concentration of $1 \mathrm{mg} / \mathrm{mL}$ for all the compounds. The stocks were stored at $-80{ }^{\circ} \mathrm{C}$ before analysis.

The calibration standard mixture was prepared by serial dilution of the standard stock solutions with methanol to yield concentrations of $0.2-25 \mu \mathrm{g} / \mathrm{mL}$ for GNAC, GNAB, and GNAD, $0.4-50 \mu \mathrm{g} / \mathrm{mL}$ for GAC2, GAD, GADM, and GLA, $0.59-75 \mu \mathrm{g} / \mathrm{mL}$ for GAG, GAB, GAA, and GAH, 0.78$100 \mu \mathrm{g} / \mathrm{mL}$ for GAF and EGL (see Table 1 for detail concentrations). 


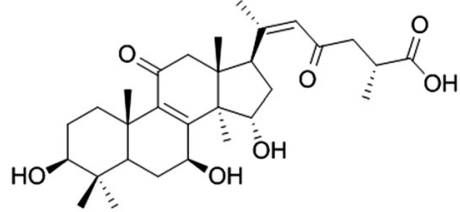

Ganoderenic Acid C 100665-42-7 $\mathrm{C}_{30} \mathrm{H}_{44} \mathrm{O}_{7}$ MW: 516.675

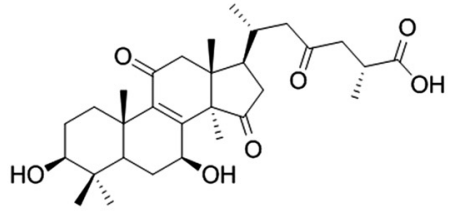

Ganoderic Acid B

$\mathrm{C}_{30} \mathrm{H}_{44} \mathrm{O}_{7}$ MW: 516.675<smiles>CC(=O)O[C@H]1C(=O)C2=C(C(=O)C[C@H]3[C@@H](C)[C@H](O)CC[C@@]23C)[C@@]2(C)C(=O)C[C@H]([C@@H](C)CC(=O)C[C@H](C)C(=O)O)[C@]12C</smiles>

Ganoderic Acid H 98665-19-1 $\mathrm{C}_{32} \mathrm{H}_{44} \mathrm{O}_{9}$ MW: 572.695<smiles>CC(=O)O[C@H]1C(=O)C2=C(C(=O)CC3C(C)(C)C(=O)CC[C@@]23C)[C@@]2(C)C(=O)C[C@H]([C@@H](C)CC(=O)CC(C)C(=O)O)[C@]12C</smiles>

Ganoderic Acid F CLQ52DLW7V $\mathrm{C}_{32} \mathrm{H}_{42} \mathrm{O}_{9}$ MW: 570.679

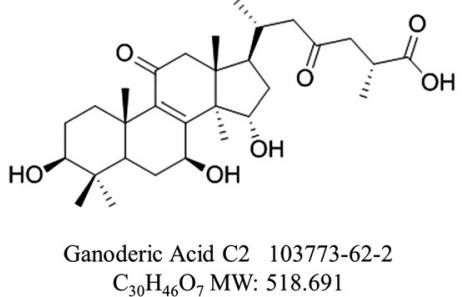

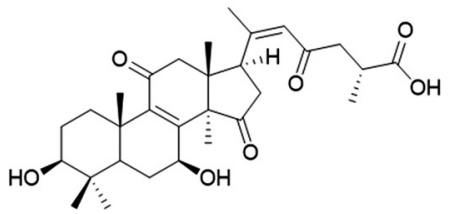

Ganoderenic Acid B 100665-41-6 $\mathrm{C}_{30} \mathrm{H}_{42} \mathrm{O}_{7}$ MW: 514.659<smiles>C/C(=C/C(=O)CC(C)C(=O)O)C1CC(=O)[C@H]2C3=C(C(=O)C[C@@]12C)[C@@]1(C)CCC(=O)C(C)(C)C1CC3O</smiles>

Ganoderenic Acid D 100665-43-8 $\mathrm{C}_{30} \mathrm{H}_{40} \mathrm{O}_{7}$ MW: 512.643<smiles>C/C(=C\CC[C@H](C)[C@H]1CC[C@]2(C)C3=C(CC[C@]12C)[C@@]1(C)CCC(=O)C(C)(C)C1CC3=O)C(=O)O</smiles>

Ganoderic Acid DM 173075-45-1 $\mathrm{C}_{30} \mathrm{H}_{44} \mathrm{O}_{4}$ MW: 468.678<smiles>CC(CC(=O)C[C@H](C)[C@H]1CC(=O)[C@H]2C3=C(C(O)CC21)[C@@]1(C)CCC(O)C(C)(C)C1C3=O)C(=O)O</smiles>

Ganoderic Acid G 98665-22-6 $\mathrm{C}_{30} \mathrm{H}_{44} \mathrm{O}_{8}$ MW: 532.674

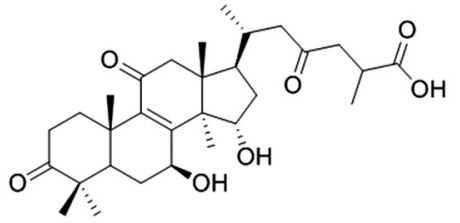

Ganoderic Acid A 81907-62-2 $\mathrm{C}_{30} \mathrm{H}_{44} \mathrm{O}_{7} \mathrm{MW}: 516.675$<smiles>CC(CC(=O)C[C@H](C)[C@H]1CC(=O)[C@H]2C3=C(C(=O)C[C@]21C)[C@@]1(C)CCC(=O)C(C)(C)C1C[C@H]3O)C(=O)O</smiles>

Ganoderic Acid D 108340-60-9 $\mathrm{C}_{30} \mathrm{H}_{42} \mathrm{O}_{7}$ MW: 514.659<smiles>C/C(=C\CC[C@H](C)[C@H]1CC[C@H]2C3=CCC4C(C)(C)C(=O)CC[C@]4(C)C3=CC[C@@]21C)CO</smiles>

Ganoderol A 104700-97-2 $\mathrm{C}_{30} \mathrm{H}_{46} \mathrm{O}_{2}$ MW: 438.696<smiles>CC(C)[C@H](C)/C=C/[C@H](C)[C@H]1CCC2C3=CC=C4C[C@@H](O)CC[C@]4(C)[C@H]3CC[C@]21C</smiles>

Fig. 1. Structure and chemical properties of the thirteen standards in Ganoderma lucidum

In addition, another set of QC samples were prepared separately at low concentration quality control (LQC), medium concentration quality control (MQC), and high concentration quality control (HQC) with the concentrations shown in Table 2.

The high performance liquid chromatography (HPLC) system consisted of an Agilent 1260 HPLC system (Agilent Technologies Inc., Santa Clara, CA, USA), equipped with a quaternary pump (G1311B), an autosampler (G7129A), an automatic thermostatic column compartment, a DAD detector (G1315D). Agilent OpenLAB CDS Chemstation Edition for LC\&LC/MS Systems (Rev C.01.07) was used to analyze the HPLC data.
The chromatographic separation was performed using an Hypersil Gold aQ Endcapped $\mathrm{C}_{18}$ reversed-phase column $(100 \mathrm{~mm} \times 2.1,1.9 \mu \mathrm{m})$ together with a $\mathrm{C}_{18}$ guard column $(10 \mathrm{~mm} \times 4.4 \mu \mathrm{m})$ with column temperature set at $35^{\circ} \mathrm{C}$. HPLC-DAD detection was used for the assay of reference compounds. The mobile phase consisted of acetonitrile (A) and $0.1 \%$ phosphoric acid $(\mathrm{v} / \mathrm{v}, \mathrm{B})$ using gradient elution (Table 2). The flow rate was $0.2 \mathrm{~mL} / \mathrm{min}$, and the detection wavelength was set at $254 \mathrm{~nm}$. An aliquot of $5 \mu \mathrm{L}$ solution was injected for acquiring HPLC chromatograms.

Product extraction. The herbal product powder was extracted using chloroform. A water bath was set up at 
Table 1. Concentrations for the standards

\begin{tabular}{|c|c|c|c|c|c|c|c|c|c|}
\hline & \multicolumn{6}{|c|}{ Standard curves } & \multicolumn{3}{|c|}{ Quality control } \\
\hline & LLOD & LLOQ & & & & & LQC & MQC & HQC \\
\hline GNAC & 0.20 & 0.39 & 1.56 & 6.25 & 12.5 & 25 & 0.78 & 3.13 & 20 \\
\hline GAC2 & 0.39 & 0.78 & 3.13 & 12.50 & 25.0 & 50 & 1.56 & 6.25 & 40 \\
\hline GAG & 0.59 & $1.17^{\circ}$ & 4.69 & 18.75 & 37.5 & 75 & 2.34 & 9.38 & 60 \\
\hline GNAB & 0.20 & 0.39 & 1.56 & 6.25 & 12.5 & 25 & 0.78 & 3.13 & 20 \\
\hline GAB & 0.59 & 1.17 & 4.69 & 18.75 & 37.5 & 75 & 2.34 & 9.38 & 60 \\
\hline GAA & 0.59 & 1.17 & 4.69 & 18.75 & 37.5 & 75 & 2.34 & 9.38 & 60 \\
\hline GAH & 0.59 & 1.17 & 4.69 & 18.75 & 37.5 & 75 & 2.34 & 9.38 & 60 \\
\hline GNAD & 0.20 & 0.39 & 1.56 & 6.25 & 12.5 & 25 & 0.78 & 3.13 & 20 \\
\hline GAD & 0.39 & 0.78 & 3.13 & 12.50 & 25.0 & 50 & 1.56 & 6.25 & 40 \\
\hline GAF & 0.78 & 1.56 & 6.25 & 25.00 & 50.0 & 100 & 3.13 & 12.50 & 80 \\
\hline GADM & 0.39 & 0.78 & 3.13 & 12.50 & 25.0 & 50 & 1.56 & 6.25 & 40 \\
\hline GLA & 0.39 & 0.78 & 3.13 & 12.50 & 25.0 & 50 & 1.56 & 6.25 & 40 \\
\hline EGL & 0.78 & 1.56 & 6.25 & 25.00 & 50.0 & 100 & 3.13 & 12.50 & 80 \\
\hline
\end{tabular}

LLOD: lower limit of detection.

LLOQ: lower limit of quantification.

LQC: low concentration quality control.

MQC: medium concentration quality control.

HQC: high concentration quality control.

Table 2. Program of HPLC gradient elution

\begin{tabular}{lcc}
\hline Time (min) & Acetonitrile (\%) & $0.1 \%$ phosphoric acid (\%) \\
\hline 0 & 10 & 90 \\
5 & 10 & 90 \\
8 & 30 & 70 \\
18 & 30 & 70 \\
23 & 45 & 55 \\
33 & 45 & 55 \\
36 & 70 & 30 \\
41 & 85 & 15 \\
46 & 85 & 15 \\
61 & 100 & 0 \\
101 & 100 & 0 \\
102 & 10 & 90 \\
120 & 10 & 90 \\
\hline
\end{tabular}

approximately $80{ }^{\circ} \mathrm{C}$. The product powder $(0.5 \mathrm{~g}$ GLSF, $0.4 \mathrm{~g}$ GLS, or $0.1 \mathrm{~g}$ GLF) was weighted precisely and put into a round bottom flask, and $12.5 \mathrm{~mL}$ of chloroform added. The mixture was set inside the water bath attached to a reflux condenser. After being refluxed for approximately $2 \mathrm{~h}$, the mixture was centrifuged at $4,000 \mathrm{rpm}$ for $10 \mathrm{~min}$. The supernatant was collected and the pellet was extracted by repeating the steps above. The supernatant collected was combined and filtered using Whatman No. 1 filter paper. The solvent was evaporated using a rotary evaporator. The resulting solid residue was re-dissolved in $5 \mathrm{~mL}$ of methanol by sonication for $30 \mathrm{~min}$. The reconstituted solution was filtered again using Whatman No. 1 filter paper. The final resulting solution was collected and stored at $-20{ }^{\circ} \mathrm{C}$ until HPLC evaluation.

Method validation. The method was validated in terms of specificity, selectivity, sensitivity, precision, accuracy, stability, and recovery according to the FDA guideline of bioanalytical assay.

Specificity and selectivity were evaluated by observing the potential interference by the impurities from the standards or working solutions. The peak resolution (Rs) between neighboring peaks was used to assess the separation efficiency and method robustness. Rs was calculated by the equation of Rs $=2\left(t_{\mathrm{R} 2}-t_{\mathrm{R} 1}\right) /\left(w_{1}+w_{2}\right)$, where $t_{\mathrm{R}}$ and $w$ are the retention time and peak width, respectively. A value greater than 1.5 is considered to be sufficient for quantitative analysis.

The linearity was assessed based on the calibration curves. Each calibration curve contained at least six different concentrations and was conducted in triplicate. Calibration curves were constructed by plotting the peak area of analyte versus the analyte concentration and fitted by weighted linear least-squares regression analysis with the weighing factor of $1 / \mathrm{X}^{2}$ ( $\mathrm{X}$ is the nominal concentration of the corresponding sample).

The lower limit of quantification (LLOQ) was defined as the concentration of each analyte where the signal-to-noise ratio is $\geq 10$ and the signal is reproducible to a precision of $20 \%$ and accuracy of $80-120 \%$.

The inter-batch precision and accuracy of the developed method were determined by validating quality control samples at LLOQ, low (LQC), median (MQC) and high (HQC) concentrations. Each sample was analyzed in five individual batches. The precision is presented as a measure of relative standard deviation (RSD) among the triplicate samples or average measured concentrations from 3 different days, while the accuracy is assessed as the percentage bias from the nominal concentration (\%).

Two batches of GLS and GLF (one for each) were used as the representative herbs for evaluating the spiked sample recovery. The standards stocks of these 13 compounds were 
spiked into $0.4 \mathrm{~g}$ of GLS or $0.1 \mathrm{~g}$ of GLF with the equivalent amount. For those compounds not detectable in the herbal extract, the LQC concentrations were used. The solvent was evaporated thoroughly in a chemical hood for $0.5 \mathrm{~h}$. Afterward, the raw herbs spiked with standards using the previous method. The content of each compound was analyzed using the same method.

The stability of the compounds was tested under the conditions of long-term storage $\left(-20{ }^{\circ} \mathrm{C}\right.$ for 1 month), short-term storage (room temperature for $24 \mathrm{~h}$ ), freeze/thaw cycle (3 times), and autosampler $\left(4{ }^{\circ} \mathrm{C}\right.$ for $\left.24 \mathrm{~h}\right)$. All the samples were then processed and analyzed together with the freshly prepared samples. All these stability tests were performed in triplicate using LQC and HQC samples. The percent deviation in concentrations was used as a parameter for stability evaluation, which was set within $15 \%$ of the nominal concentration.

HPLC fingerprinting. The HPLC-DAD assay was utilized to analyze GL products, including standard GLF, standard GLS, their mixture (GLS: GLF $=4: 1$, based on weight), 14 batches of GLF, 9 batches of GLS, and 5 batches of GLSF as well. The samples were prepared using the method described in Section 2.2.2. The solution was transferred to a sample injection vial and $20 \mu \mathrm{L}$ was injected into the HPLC-DAD system for analysis.

Data analysis was performed by professional software named Similarity Evaluation System for Chromatographic Fingerprint of Traditional Chinese Medicine (Version 2004 A), which was developed and recommended by the Chinese State Food and Drug Administration. In this study, the software was employed to synchronize and conduct quantitative comparisons among different samples. It is also used to compute and generate the mean chromatogram as a representative standard fingerprint chromatogram for a group of chromatograms. The similarity value of each chromatogram was calculated based on the cosine value for the vectorial angle.

Principal component analysis. The inter-batch variability of GLS and GLF was further assessed using principal component analysis (PCA), which was conducted in R ( $\mathrm{v} 4.03$, https://www.r-project.org/) with a package of "cluster." The amount of the major components were read into $\mathrm{R}$ for further process. For GLF, all the components were included, while only ten compounds (excluding GNAC, GAC2, and GLA) for GLS since the amount is too low.

The two most dominant principal components (PC) were used for PCA plotting. Hierarchical cluster analysis (HCA) was then processed based on the results of PCA plot.

\section{RESULTS}

\section{HPLC assay development and validation}

The constitution of the mobile phase was firstly optimized. Because some of the active components are weak acids, phosphoric acid was used to make an acidic buffer $(0.1 \%, \mathrm{v} /$ v) to reduce the ionization of these compounds for better chromatographic resolution. Different elution conditions with acetonitrile and phosphoric acid $(0.1 \%)$ were tested and compared. Because of the great difference in the polarity of these compounds, gradient elution was applied. The elution conditions were optimized which was able to separate these 13 compounds in $124 \mathrm{~min}$ (Table 3).

The column temperature was an important parameter influencing resolution and selectivity. Room temperature (around $25{ }^{\circ} \mathrm{C}$ ), $30{ }^{\circ} \mathrm{C}$ and $35{ }^{\circ} \mathrm{C}$ were tested. The results indicated that $30{ }^{\circ} \mathrm{C}$ was the most suitable for the best resolution.

The selection of detection wavelength was essential for detecting the components of GL products, and thus it is important for establishing the HPLC fingerprint. Diodearray detector (DAD) was applied to select the optimal wavelength by testing four conditions including 210, 230, 254, and $360 \mathrm{~nm}$. The results indicated that $254 \mathrm{~nm}$ were able to generate the most abundant peaks when compared to other wavelengths.

The representative chromatograms of LLOQ, LQC, MQC, and HQC samples are shown in Fig. 2A-D. The typical retention time is shown in Table 3. These results suggested that the thirteen compounds at high and low concentrations could be clearly detected. No interfering peaks at the retention time were observed for all the, which suggested a desirable specificity and selectivity.

The Rs results are shown in Table 3, which are all greater than 1.5, except for GAA and GAH, which is 1.1 (Table 3). Many approaches have been examined to improve the separation, including change of column, modification of the mobile phase and elution gradient, temperature, etc. None of them could further increase the Rs due to the extremely similar chemical structure of these two compounds. However, GAA and GAH were able to be resolved under current conditions with acceptable accuracy and precision. Therefore, we believe this method still be able to be used for quantification.

Table 3. Retention time and peak resolution of neighboring peaks of the compounds in HPLC chromatogram

\begin{tabular}{lccc}
\hline $\begin{array}{l}\text { Peak } \\
\text { Resolution }\end{array}$ & $\begin{array}{c}\text { Retention time } \\
\left(t_{R}\right)\end{array}$ & $\begin{array}{c}\text { Peak width } \\
(w)\end{array}$ & $\begin{array}{c}\text { Peak resolution } \\
(R s)\end{array}$ \\
\hline GNAC & 19.591 & 0.3 & \\
GAC2 & 20.518 & 0.4 & 2.6 \\
GAG & 22.866 & 0.55 & 4.9 \\
GNAB & 23.430 & 0.3 & 1.3 \\
GAB & 24.263 & 0.5 & 2.1 \\
GAA & 26.126 & 0.3 & 4.7 \\
GAH & 26.465 & 0.33 & 1.1 \\
GNAD & 27.600 & 0.2 & 4.3 \\
GAD & 28.062 & 0.35 & 1.7 \\
GAF & 29.563 & 0.125 & 6.3 \\
GADM & 44.265 & 0.3 & 69.2 \\
GLA & 56.140 & 0.35 & 36.5 \\
EGL & 96.068 & 2.9 & 24.6 \\
\hline
\end{tabular}



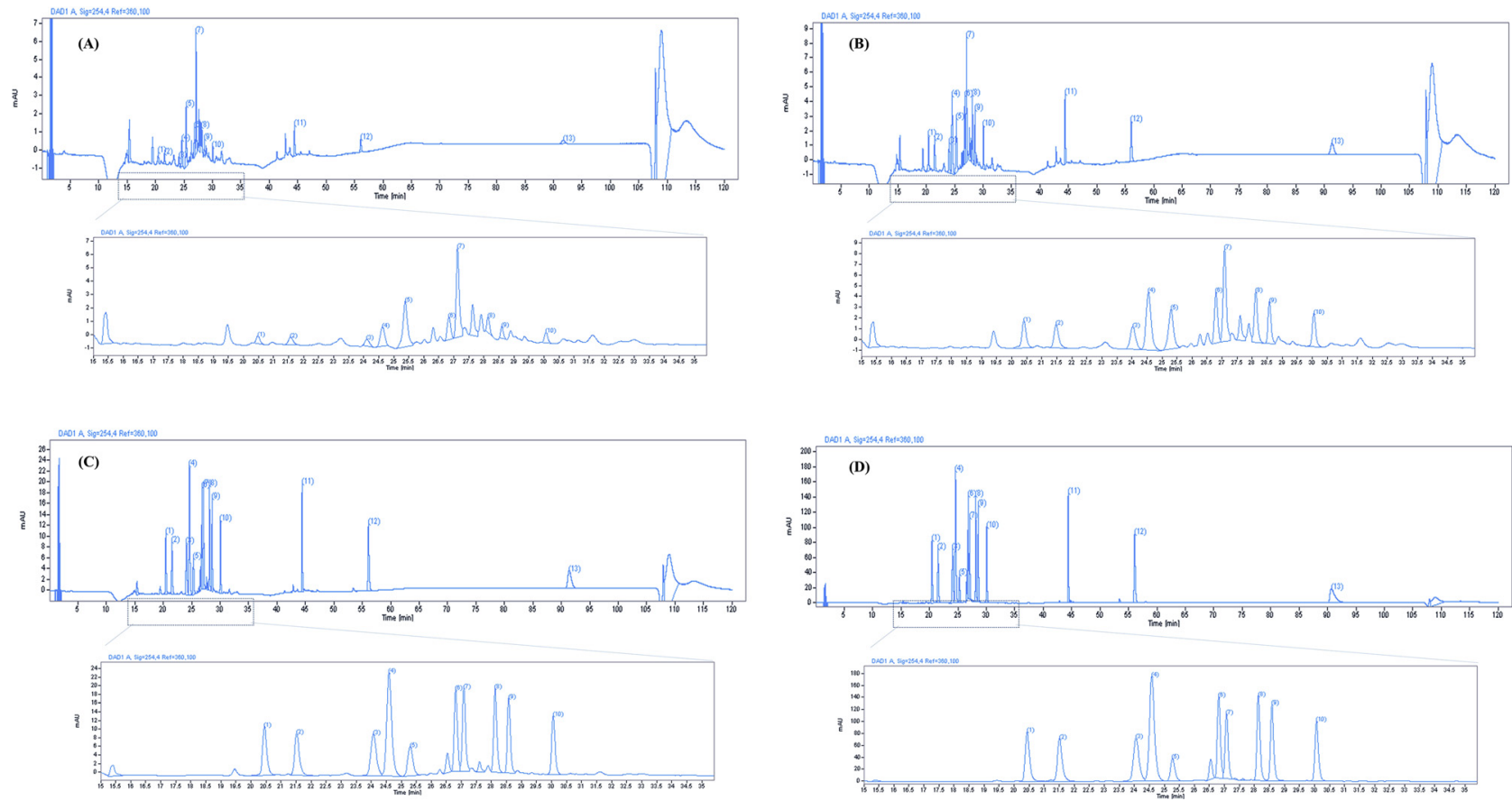

Fig. 2. Chromatograms of standard samples at concentrations of (A) LLOQ, (B) LQC, (C) MQC, and (D) HQC

The calibration curve was found linear over concentrations of $0.3125-20 \mu \mathrm{g} / \mathrm{mL}$ for GNAC, GAA, GAH, and GNAD; $0.78125-50 \mu \mathrm{g} / \mathrm{mL}$ for GAC2, GAG, GAB, and GAF; $0.15625-10 \mu \mathrm{g} / \mathrm{mL}$ for GNAB, GAD, and GADM; $0.625-40 \mu \mathrm{g} / \mathrm{mL}$ for GRA; and $1.5625-100 \mu \mathrm{g} / \mathrm{mL}$ for EGL. The correlation coefficient $\left(R^{2}\right)$ was calculated to be greater than 0.99 for these standard curves.

The lower limit of quantification (LLOQ) was $0.3125 \mu \mathrm{g} /$ $\mathrm{mL}$ for GNAC, GAA, GAH, and GNAD; $0.78125 \mu \mathrm{g} / \mathrm{mL}$ for
GAC2, GAG, GAB, and GAF; $0.15625 \mu \mathrm{g} / \mathrm{mL}$ for GNAB, GAD, and GADM; $0.625 \mu \mathrm{g} / \mathrm{mL}$ for GRA; and $1.5625 \mu \mathrm{g} / \mathrm{mL}$ for EGL.

The inter-batch accuracy and precision were determined by measuring five replicates of QC samples at four concentration levels (LLOQ, LQC, MQC, and HQC) on separate batches. The results are shown in Table 4. The intraday accuracy was within $87.0-114.6 \%$, while the precision was within $11.96 \%$ for all the samples tested.

Table 4. Precision and accuracy of HPLC assay for the standards

\begin{tabular}{|c|c|c|c|c|c|c|c|}
\hline Comp. & Levels & Precision (\%) & Accuracy (\%) & Comp. & Levels & Precision (\%) & Accuracy (\%) \\
\hline \multirow[t]{3}{*}{ GANC } & LLOQ & 1.13 & $97.9-100.5$ & \multirow[t]{3}{*}{ GAND } & LLOQ & 1.25 & $97.3-100.4$ \\
\hline & LQC & 3.94 & $98.3-109.6$ & & LQC & 4.45 & $98.9-112.0$ \\
\hline & MQC & 4.45 & $96.7-106.9$ & & MQC & 3.47 & $98.6-105.7$ \\
\hline \multirow[t]{4}{*}{ GAC2 } & LLOQ & 1.52 & $96.7-100.8$ & \multirow[t]{4}{*}{ GAD } & LLOQ & 1.47 & $94.1-97.4$ \\
\hline & LQC & 5.99 & $98.0-114.9$ & & LQC & 11.96 & $93.8-111.5$ \\
\hline & MQC & 3.43 & $96.0-104.7$ & & MQC & 2.84 & $101.2-109.1$ \\
\hline & HQC & 4.49 & $93.4-103.3$ & & HQC & 3.56 & $90.4-98.4$ \\
\hline \multirow{3}{*}{ GAG } & LQC & 5.84 & $98.3-104.9$ & \multirow{3}{*}{ GAF } & LQC & 5.24 & $90.7-103.6$ \\
\hline & MQC & 3.66 & $98.9-108.0$ & & MQC & 10.18 & $94.1-102.7$ \\
\hline & HQC & 4.47 & $93.4-103.3$ & & HQC & 7.17 & $87.0-105.7$ \\
\hline \multirow[t]{4}{*}{ GAB } & LLOQ & 1.53 & $96.9-99.6$ & \multirow[t]{4}{*}{ GADM } & LLOQ & 1.09 & $98.5-101.4$ \\
\hline & LQC & 5.95 & $96.9-113.3$ & & LQC & 6.29 & $86.5-102.6$ \\
\hline & MQC & 3.65 & $99.5-108.6$ & & MQC & 3.04 & $98.5-105.3$ \\
\hline & HQC & 2.87 & $93.5-101.1$ & & HQC & 3.30 & $96.2-102.8$ \\
\hline \multirow[t]{3}{*}{ GNAB } & LLOQ & 1.60 & $95.4-99.8$ & \multirow[t]{3}{*}{ GRA } & LLOQ & 1.22 & $97.3-100.4$ \\
\hline & LQC & 6.84 & $99.0-107.0$ & & LQC & 4.19 & $99.2-111.3$ \\
\hline & HQC & 5.41 & $89.5-103.3$ & & HQC & 4.73 & $94.0-103.4$ \\
\hline \multirow[t]{4}{*}{ GAA } & LLOQ & 2.22 & $94.6-100.3$ & \multirow[t]{4}{*}{ EGL } & LLOQ & 0.76 & $97.5-98.5$ \\
\hline & LQC & 4.52 & $100.7-112.5$ & & LQC & 54.66 & $82.9-111.3$ \\
\hline & MQC & 3.87 & $99.2-107.3$ & & MQC & 17.18 & $98.8-104.4$ \\
\hline & HQC & 5.11 & $93.7-102.9$ & & HQC & 7.24 & $94.8-113.9$ \\
\hline \multirow[t]{4}{*}{ GAH } & LLOQ & 1.34 & $97.1-100.3$ & & & & \\
\hline & LQC & 4.30 & $99.7-112.5$ & & & & \\
\hline & MQC & 6.67 & $97.1-114.6$ & & & & \\
\hline & HQC & 3.92 & $93.7-103.7$ & & & & \\
\hline
\end{tabular}


Table 5. Stability test of the 13 compounds

\begin{tabular}{|c|c|c|c|c|c|c|c|c|}
\hline \multirow[b]{2}{*}{ Compounds } & \multicolumn{2}{|c|}{$\begin{array}{c}\text { Freeze-Thaw cycle ( } 3 \\
\text { cycles) }\end{array}$} & \multicolumn{2}{|c|}{ Autosampler (24 h) } & \multicolumn{2}{|c|}{$\begin{array}{l}\text { Room temperature } \\
\qquad(24 \mathrm{~h})\end{array}$} & \multicolumn{2}{|c|}{ Long term $\left(-20^{\circ} \mathrm{C}\right)$} \\
\hline & LQC & HQC & LQC & HQC & LQC & HQC & LQC & HQC \\
\hline GNAC & 107.5 & 105.1 & 114.4 & 103.7 & 101.9 & 100.4 & 101.1 & 103.9 \\
\hline GAC2 & 99.3 & 105.0 & 110.2 & 103.7 & 102.2 & 100.8 & 108.3 & 103.9 \\
\hline GAG & 106.2 & 98.8 & 105.4 & 104.0 & 101.8 & 100.6 & 104.6 & 103.9 \\
\hline GAB & 112.7 & 95.8 & 114.4 & 104.1 & 102.8 & 99.4 & 104.1 & 104.0 \\
\hline GNAB & 97.7 & 104.5 & 106.5 & 104.0 & 101.4 & 100.4 & 101.0 & 103.7 \\
\hline GAA & 102.6 & 103.0 & 106.3 & 105.5 & 105.6 & 100.7 & 101.9 & 103.7 \\
\hline GAH & 103.4 & 100.3 & 101.5 & 104.7 & 108.1 & 100.9 & 101.0 & 103.5 \\
\hline GNAD & 93.4 & 102.4 & 116.8 & 103.9 & 101.0 & 99.9 & 95.2 & 104.3 \\
\hline GAD & 96.8 & 101.8 & 108.5 & 104.2 & 102.4 & 100.8 & 101.5 & 104.0 \\
\hline GAF & 95.0 & 96.7 & 106.5 & 104.3 & 104.2 & 100.8 & 102.5 & 103.7 \\
\hline GADM & 101.2 & 99.1 & 101.2 & 103.9 & 103.8 & 100.7 & 100.4 & 104.1 \\
\hline GRA & 100.1 & 102.1 & 110.4 & 104.8 & 103.0 & 100.7 & 101.1 & 104.3 \\
\hline EGL & 85.6 & 102.8 & 108.3 & 104.5 & 104.8 & 101.8 & 114.3 & 108.2 \\
\hline
\end{tabular}

Table 6. Content in GLS chloroform extract (standard, and 10 batches) (unit: $\mathrm{mg} / \mathrm{g}$ )

\begin{tabular}{|c|c|c|c|c|c|c|c|c|c|c|}
\hline \multirow[b]{2}{*}{ Comp. } & \multirow[b]{2}{*}{ Standard } & Batch 1 & Batch 2 & Batch 3 & Batch 4 & Batch 5 & Batch 6 & Batch 7 & Batch 8 & Batch 9 \\
\hline & & \multicolumn{2}{|c|}{2015} & \multicolumn{3}{|c|}{2016} & \multicolumn{2}{|c|}{2017} & \multicolumn{2}{|c|}{2018} \\
\hline GNAC & 0.00993 & & & & & & & & & \\
\hline GAC2 & 0.00091 & & & & & & & & & \\
\hline GAG & 0.00659 & & 0.00354 & & & & & & & \\
\hline GNAB & 0.00052 & & 0.00185 & & & & & & & \\
\hline GAB & 0.00132 & 0.00104 & 0.00000 & & & 0.00000 & 0.00147 & 0.00225 & 0.00230 & 0.00099 \\
\hline GAA & 0.00279 & & & & & & & 0.01894 & & \\
\hline GAH & 0.00366 & 0.01748 & 0.01798 & 0.01617 & 0.01643 & 0.01551 & 0.01451 & 0.01289 & 0.01597 & 0.00935 \\
\hline GNAD & 0.00072 & 0.00302 & 0.00184 & & & 0.00167 & & 0.00481 & & \\
\hline GAD & 0.01232 & 0.01440 & 0.01625 & & & 0.01409 & & & & \\
\hline GAF & 0.00304 & 0.00667 & 0.00497 & & & 0.00515 & & & & \\
\hline GADM & 0.00555 & 0.00985 & 0.00829 & 0.01028 & 0.01155 & 0.00654 & 0.03739 & 0.03863 & 0.04143 & 0.03304 \\
\hline GLA & & & & & & & & & & \\
\hline EGL & 0.00198 & 0.95622 & 0.77373 & 0.85249 & 0.91375 & 0.82908 & 0.89735 & 1.09360 & 1.18982 & 0.60873 \\
\hline
\end{tabular}

The stability of the 12 compounds after 3 cycles of freeze-thaw, 24 hours in the autosampler, and 48 hours at room temperature was tested (see Table 5). At high concentration (HQC), all the samples are stable with deviation from the control samples less than $15 \%$.

The spiked sample recovery was ranged from 69 to $119 \%$, 79 to $115 \%$, and 85 to $105 \%$ for samples of GLS, GLF, and GLSF, respectively.

\section{Quantification of the active components}

The concentration of the thirteen compounds was determined using the standard curves, and their content in the herbal extracts was calculated. The results are shown in Tables 6-8 and Fig. 3. Among the 13 standards, GLA was only detectable in the standard GLF sample with a very low quantity, but not in any other samples indicating that GLA may not be the main component in GLS or GLF samples (Tables 6-8). It may only play a minor role in the activity of GL products.

EGL was found in all the GLS samples in a consistent amount (average of $0.9 \mathrm{mg} / \mathrm{g}$ with the relative standard deviation (RSD) less than 20\%). However, EGL was very low in the standard GLS sample. The possible reason is the commercial products were processed samples while the standard GLS is the raw material without processing. So the extraction of EGL in the standard GLS may not be sufficient. In contrast, EGL showed a large variance of existence in GLF samples. It was only detectable in the standard GLF and 4 out of 14 batches of GLF samples (Table 7).

The results showed that the abundance of these compounds was much higher in the extractions of the fruiting body than that of spore samples, except EGL (GLS is 4.5 higher than GLF extracts, average $0.9 \mathrm{mg} / \mathrm{g}$ in GLS versus 0.2 $\mathrm{mg} / \mathrm{g}$ in GLF). GNAC and GAC2 were not detectable in GLS, while the mean contents in GLF were 0.11 and 0.42 $\mathrm{mg} / \mathrm{g}$ in GLF samples). The remaining compounds were identified with low amounts in the GLS samples (less than $0.02 \mathrm{mg} / \mathrm{g})$, but substantial contents were detected in GLF extracts $(0.07-1.17 \mathrm{mg} / \mathrm{g})$.

Large variations were identified with RSD greater than $60 \%$ among the GLF samples (See Fig. 4), indicating significant variability in the chemical compositions. For the 


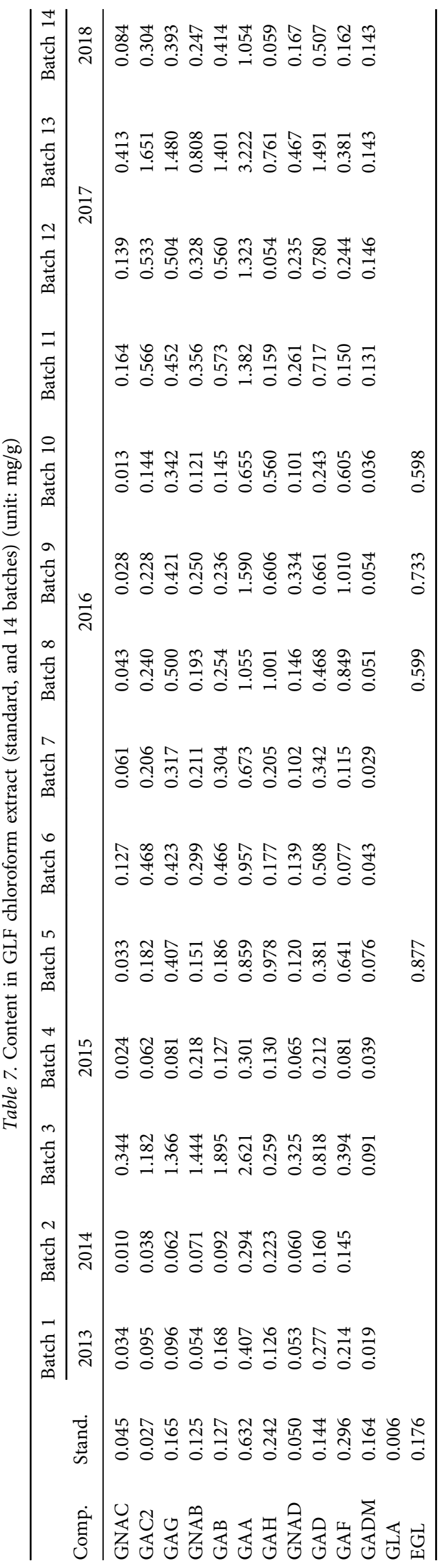

GLS extracts, only compounds of GADM and EGL were constantly detectable in these samples, while others could only be quantified in a few of these samples leading to sparse data available. Therefore, only the RSD of GADM and EGL were calculated, which are 65 and $18.9 \%$ for GADM and EGL, respectively.

The sample mixture of GLSF (GLS: GLF $=4: 1, \mathrm{w} / \mathrm{w}$ ) was also analyzed using the validated HPLC-DAD method. As expected, the amount of compounds identified was greater than GLS but lower than GLF. In addition, a large variation was observed for most of the compounds as well (Table 8).

\section{HPLC fingerprint}

The chromatographic fingerprints were generated for 14 batches of GLF, 9 batches of GLS, and their standard samples. Before analysis, the chromatograms were modified by excluding the solvent peak as well as the peaks after $60 \mathrm{~min}$. Although the retention time for EGL was found $\sim 95 \mathrm{~min}$, it was excluded for further data analysis. The retention time was found much longer compared with other peaks. Such a long time for elution tends to result in unstable retention time (e.g., 5\% variation may cause more than 5-min difference), so it is not suitable for similarity analysis. In terms of specificity, EGL is a common component found in many yeasts and other fungal membranes (with a similar function to cholesterol) [20]. It is not unique for GL samples. Therefore it is not a typical chemical marker for GLS and GLF products. Therefore, only the Ganoderma acids and GLA were included for the fingerprint construction.

Two simulative median fingerprint chromatograms were then produced for GLS and GLF (without standard samples). The similarity values of each chromatograph against the corresponding simulative median fingerprint chromatograms, as well as their inter-batch difference, was calculated (Tables 9 and 10).

As shown in Fig. 5, the chemical profiles of 9 batches of GLS samples were not consistent. The similarity results showed these samples could be partitioned into 2 major groups (year 2015 and 2016 versus 2017 and 2018). It appeared that samples collected in 2015 and 2016 have similar quality (similarity values from $0.85-0.92$ ), while samples in 2017 and 2018 are close to each other (0.960.99). The results indicated the quality of GLS is highly affected by the year of collection. All these TRT GLS products are not consistent with the standard GLS with similarity values less than 0.5 .

The chromatograms of 14 batches of GLF are shown in Fig. 6. Similar to the GLS samples, the quality of GLF samples are not consistent according to the similarity analysis. The samples collected in the years 2013 and 2014 had similar chemical profiles $(>0.7)$. The samples collected in years 2015 and 2016 exhibited similar quality as well except batch 11, which was considered to be an outlier since it was quite different from any other samples regardless of the year collected. The more recent samples 
Table 8. Content in GLSF chloroform extract (standard mixture, and 5 batches, GLS: GLF = 4:1) (unit: mg/g)

\begin{tabular}{|c|c|c|c|c|c|c|}
\hline Compound & Standard mixture ${ }^{\mathrm{a}}$ & Batch 1 & Batch 2 & Batch 3 & Batch 4 & Batch 5 \\
\hline GNAC & 0.0212 & 0.0170 & 0.0140 & 0.0056 & 0.0170 & 0.0055 \\
\hline GAC2 & 0.1003 & 0.0612 & 0.0388 & 0.0174 & 0.0801 & 0.0132 \\
\hline GAG & 0.0840 & 0.0569 & 0.0545 & 0.0226 & 0.0905 & \\
\hline GNAB & 0.0579 & 0.0382 & 0.0539 & 0.0373 & 0.0387 & \\
\hline GAB & 0.1131 & 0.0712 & 0.0806 & 0.0335 & 0.0911 & 0.0280 \\
\hline GAA & 0.2370 & 0.1459 & 0.1229 & 0.0804 & 0.1980 & 0.0695 \\
\hline GAH & 0.0540 & 0.0283 & 0.0544 & 0.0307 & 0.0446 & 0.0269 \\
\hline GNAD & 0.0356 & 0.0232 & 0.0220 & 0.0167 & 0.0217 & 0.0132 \\
\hline GAD & 0.1414 & 0.0825 & 0.0575 & 0.0521 & 0.0965 & 0.0467 \\
\hline GAF & 0.0086 & 0.0138 & 0.0418 & 0.0230 & 0.0732 & 0.0185 \\
\hline GADM & 0.0290 & 0.0072 & 0.0132 & 0.0103 & 0.0074 & 0.0073 \\
\hline \multicolumn{7}{|l|}{ GLA } \\
\hline EGL & 0.4940 & 0.5660 & 0.7094 & & & \\
\hline
\end{tabular}

${ }^{\mathrm{a}} \mathrm{Standard}$ mixture: made by mixing the standard extracts of GLS and GLF in a ratio of 4:1.
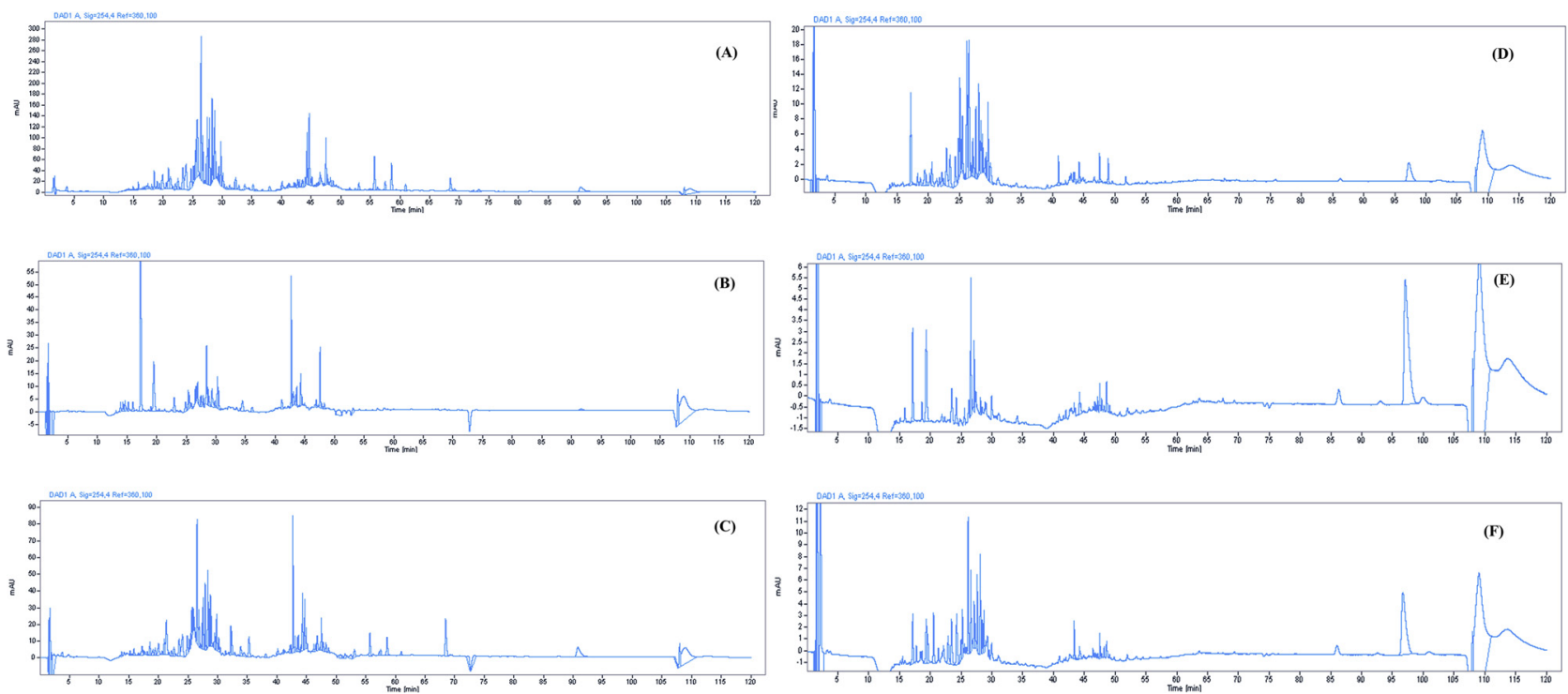

Fig. 3. Chromatograms of (A) standard GLF, (B) standard GLS, (C) standard GLSF mixture (4:1, w/w), (D) commercial products of GLF, (E) GLS, and (F) GLSF mixture (4:1, w/w)

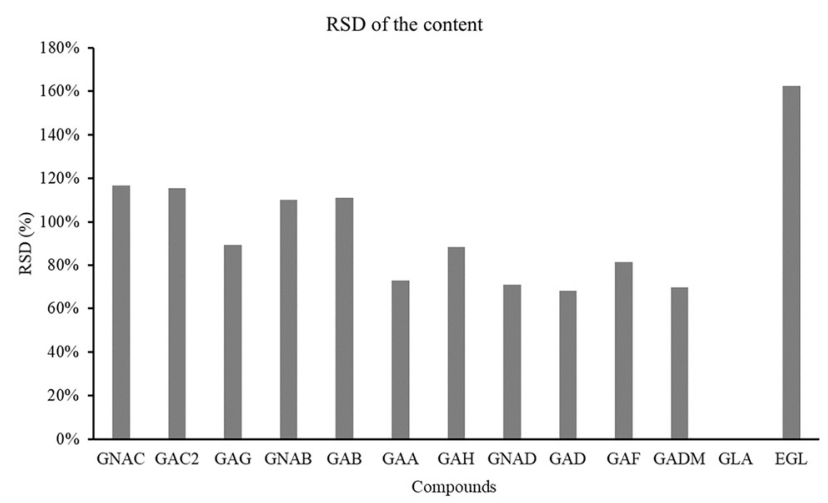

Fig. 4. Variation of the amount of 13 compounds in GLF chloroform extracts (GLA was not detectable) (years of 2017 and 2018) shared similar profiles indicating similar quality.

The results showed that the collection time has a great impact on the chemical compositions of the GL products (both fruiting body and spore), although they were from the same sites. Therefore, monitoring the chemical profiles would be important to ensure quality consistency.

None of these commercial samples provided by TRT had a similar chemical composition as the standard GL spore or fruiting body samples. Since the commercial products recruited in this study are all processed samples, it is highly likely to have different chemical fingerprints as the standards.

In this study, a marketed product (GLSF) was also tested and showed similar profiles in chemical components with a similarity value greater than 0.77 (Fig. 7). 
Table 9. Cross comparison of similarity values of GLS samples

\begin{tabular}{|c|c|c|c|c|c|c|c|c|c|c|c|}
\hline \multirow[b]{2}{*}{ Batch } & Batch 1 & Batch 2 & Batch 3 & Batch 4 & Batch 5 & Batch 6 & Batch 7 & Batch 8 & Batch 9 & Standard GLS & $\mathrm{SMC}^{\mathrm{a}}$ \\
\hline & \multicolumn{2}{|c|}{2015} & \multicolumn{3}{|c|}{2016} & \multicolumn{2}{|c|}{2017} & \multicolumn{2}{|c|}{2018} & & \\
\hline Batch 1 & 1 & & & & & & & & & & \\
\hline Batch 2 & 0.922 & 1 & & & & & & & & & \\
\hline Batch 3 & 0.850 & 0.921 & 1 & & & & & & & & \\
\hline Batch 4 & 0.911 & 0.877 & 0.798 & 1 & & & & & & & \\
\hline Batch 5 & 0.855 & 0.893 & 0.877 & 0.771 & 1 & & & & & & \\
\hline Batch 6 & 0.011 & 0.008 & 0.013 & 0.033 & 0.022 & 1 & & & & & \\
\hline Batch 7 & 0.029 & 0.03 & 0.031 & 0.048 & 0.039 & 0.992 & 1 & & & & \\
\hline Batch 8 & 0.028 & 0.031 & 0.033 & 0.046 & 0.038 & 0.979 & 0.979 & 1 & & & \\
\hline Batch 9 & 0.025 & 0.027 & 0.023 & 0.036 & 0.026 & 0.964 & 0.965 & 0.966 & 1 & & \\
\hline Standard GLF & 0.320 & 0.313 & 0.308 & 0.202 & 0.46 & 0.079 & 0.089 & 0.079 & 0.048 & 1 & \\
\hline SMC & 0.328 & 0.328 & 0.318 & 0.296 & 0.357 & 0.862 & 0.871 & 0.866 & 0.865 & 0.441 & 1 \\
\hline
\end{tabular}

${ }^{\mathrm{a} S M C}$ : Simulative Median Chromatogram.

Table 10. Cross comparison of similarity values of GLF samples

\begin{tabular}{|c|c|c|c|c|c|c|c|c|c|c|c|c|c|c|c|c|}
\hline & Batch 1 & Batch 2 & Batch 3 & Batch 4 & Batch 5 & Batch 6 & Batch 7 & Batch 8 & Batch 9 & Batch 10 & Batch 11 & Batch 12 & Batch 13 & Batch 14 & Standard $\mathrm{H}$ & $\mathrm{SMC}^{\mathrm{a}}$ \\
\hline & 2013 & 2014 & & 2015 & & \multicolumn{6}{|c|}{2016} & \multicolumn{2}{|c|}{2017} & 2018 & & \\
\hline Batch 1 & 1 & & & & & & & & & & & & & & & \\
\hline Batch 2 & 0.733 & 1 & & & & & & & & & & & & & & \\
\hline Batch 3 & 0.589 & 0.504 & 1 & & & & & & & & & & & & & \\
\hline Batch 4 & 0.847 & 0.770 & 0.665 & 1 & & & & & & & & & & & & \\
\hline Batch 5 & 0.589 & 0.537 & 0.664 & 0.530 & 1 & & & & & & & & & & & \\
\hline Batch 6 & 0.834 & 0.694 & 0.851 & 0.815 & 0.638 & 1 & & & & & & & & & & \\
\hline Batch 7 & 0.884 & 0.763 & 0.781 & 0.909 & 0.609 & 0.939 & 1 & & & & & & & & & \\
\hline Batch 8 & 0.636 & 0.548 & 0.686 & 0.546 & 0.966 & 0.685 & 0.641 & 1 & & & & & & & & \\
\hline Batch 9 & 0.587 & 0.577 & 0.775 & 0.53 & 0.813 & 0.766 & 0.687 & 0.848 & 1 & & & & & & & \\
\hline Batch 10 & 0.501 & 0.482 & 0.686 & 0.436 & 0.88 & 0.623 & 0.573 & 0.884 & 0.912 & 1 & & & & & & \\
\hline Batch 11 & 0.034 & 0.084 & 0.042 & 0.052 & 0.142 & 0.048 & 0.046 & 0.154 & 0.119 & 0.116 & 1 & & & & & \\
\hline Batch 12 & 0.035 & 0.106 & 0.048 & 0.053 & 0.127 & 0.053 & 0.050 & 0.142 & 0.117 & 0.115 & 0.964 & 1 & & & & \\
\hline Batch 13 & 0.049 & 0.100 & 0.056 & 0.063 & 0.124 & 0.052 & 0.049 & 0.145 & 0.103 & 0.101 & 0.927 & 0.922 & 1 & & & \\
\hline Batch 14 & 0.034 & 0.095 & 0.046 & 0.053 & 0.121 & 0.128 & 0.140 & 0.151 & 0.113 & 0.113 & 0.853 & 0.86 & 0.944 & 1 & & \\
\hline Standard $\mathrm{H}$ & 0.091 & 0.107 & 0.092 & 0.146 & 0.179 & 0.062 & 0.060 & 0.182 & 0.15 & 0.165 & 0.704 & 0.717 & 0.706 & 0.639 & 1 & \\
\hline SMC & 0.296 & 0.31 & 0.342 & 0.325 & 0.424 & 0.363 & 0.355 & 0.443 & 0.413 & 0.406 & 0.845 & 0.85 & 0.862 & 0.81 & 0.893 & 1 \\
\hline
\end{tabular}

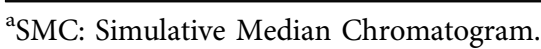




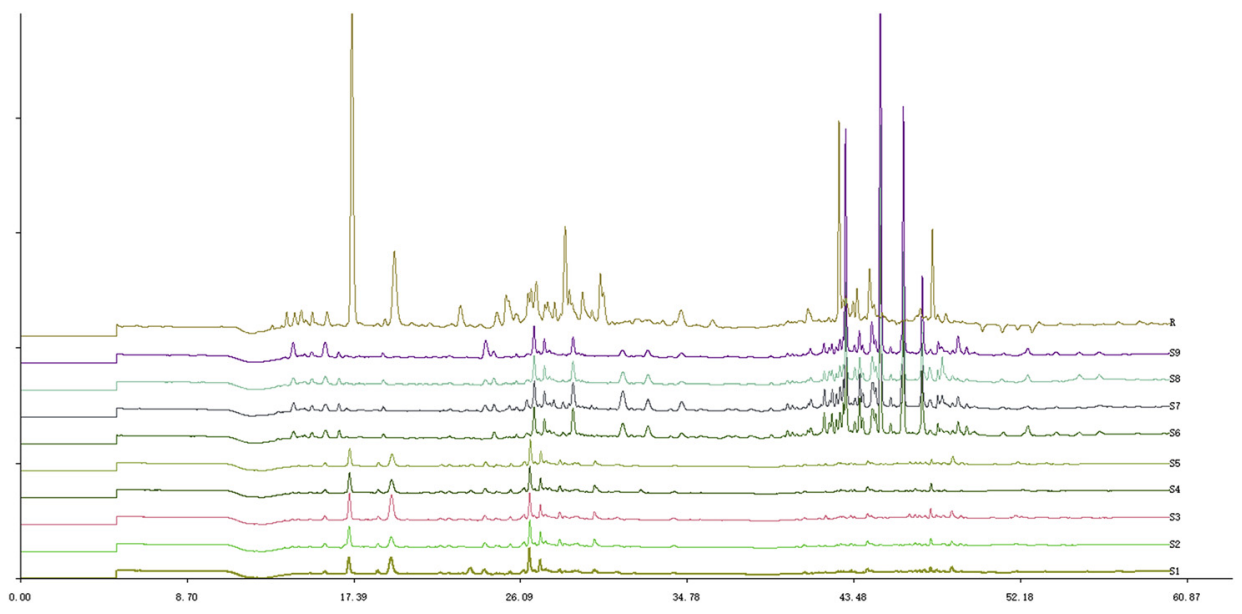

Fig. 5. HPLC fingerprints of the major chemical constituents of 10 batches of GLS sample and the standard GLS extract (S1 to S9 are batches 1 to 9 ; $\mathrm{R}$ is the standard GLS)

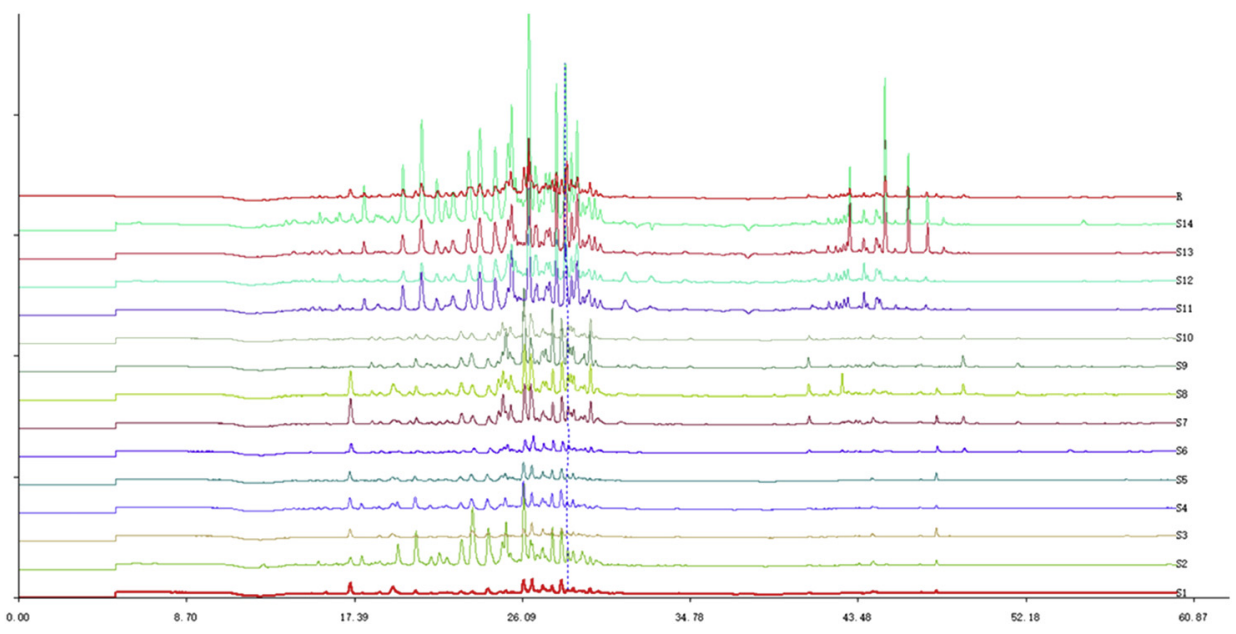

Fig. 6. HPLC fingerprints of the major chemical constituents of 14 batches of GLF samples and the standard GLF extract (S1 to S14 are batches 1 to 14 ; $\mathrm{R}$ is the standard GLF)

\section{Principal component analysis}

The PCA plots of GLS and GLF with their corresponding cluster dendrograms are shown in Fig. 8. For both GLS and GLF, the two PCs cumulatively explain $63.5 \%$ and $80.2 \%$ of the variance in the samples, which appeared to be sufficient to explain most of the variability in these products. The results are consistent with the similarity analysis, which also showed great variance in the chemical composition of GLF and GLS products. In general, the products harvested in similar years are more close to each other, although there are some outliers (such as GLF Batch 3 and 13; GLS Batch 5 and 7). HCA results showed that the quality of GLS and GLF products before 2017 are more closely related than that of after 2017. However, due to the small number of batches, the PCA and HCA results need to be further investigated.

\section{DISCUSSION}

The current study established a validated HPLC-DAD method to quantify 13 chemicals in GLS and GLF extracts.
The results indicated the content of these compounds varied a lot in both the GLS or GLF extracts. These variations were not only observed among TRT products but also found when comparing the TRT products to the standard herbs.

Except for EGL, the other 12 compounds are weak acids, so their water solubility should be low at physiological $\mathrm{pH}$ conditions. All these compounds in relatively hydrophobic with $\log \mathrm{P}$ values around 2 (predicted by using SciFinder). EGL is extremely hydrophobic with $\log$ P greater than 9. In our studies, methanol was also tried as an extraction solvent, but chloroform was proven to be more efficient in yielding rate. Therefore, chloroform was selected as the preferred solvent to extract raw herbs for quantification.

According to the chromatographic performance of EGL and its content in GLS and GLF samples, we recommend not including it for the quality analysis due to the long elution time, which may lead to variation in its retention time. Moreover, EGL is not a unique marker for GLS or GLF samples. It is a ubiquitous ingredient in many types of fungi. In addition to EGL, GLA was found in a 


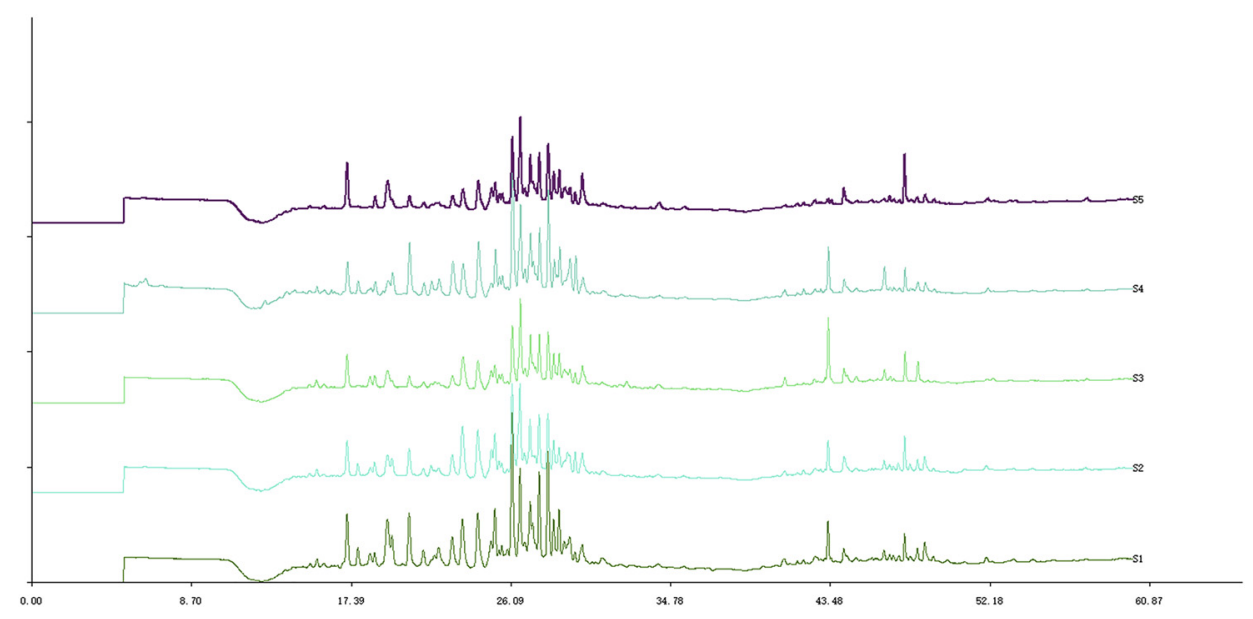

Fig. 7. HPLC fingerprints of the major chemical constituents of 6 batches of GLSF samples (GLS:GLF $=4: 1)$ (S1 to S5 are batches $1-5)$

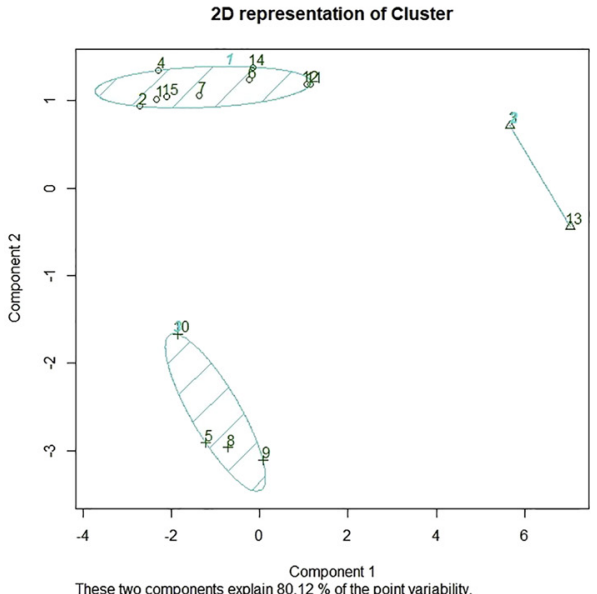

(A)

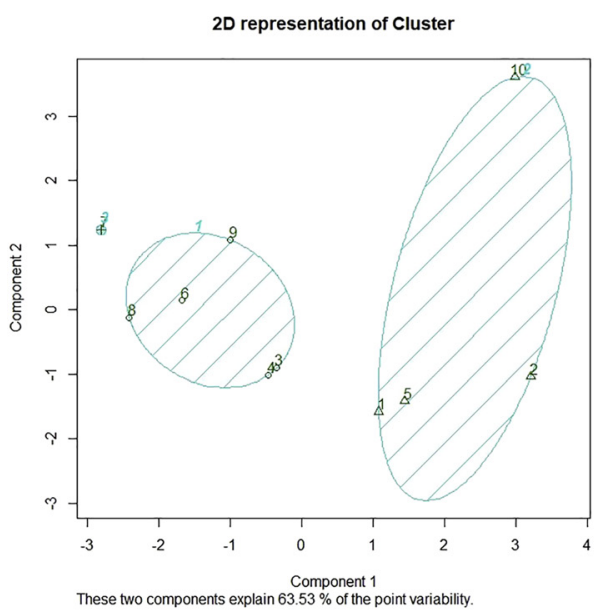

(C)

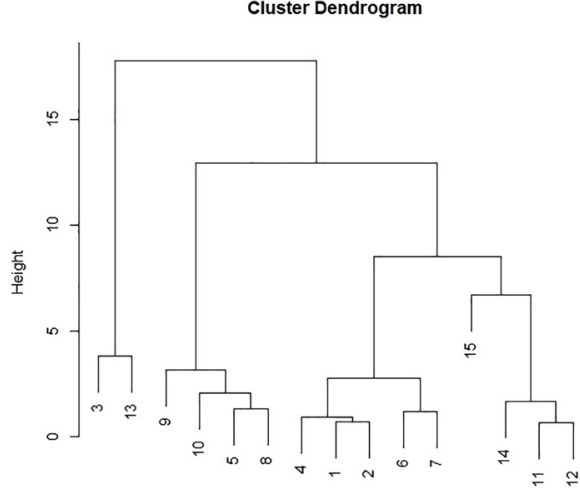

GLF

(B)

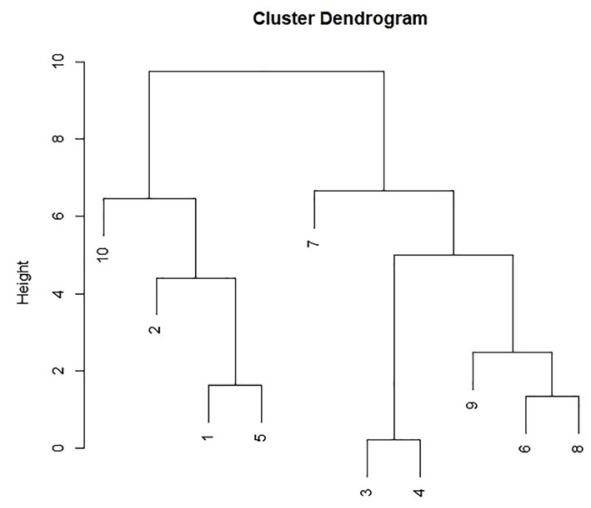

GLS

(D)

Fig. 8. PCA and HCA plots of the HPLC quantification data of GLS and GLF. (a) PCA of GLF; (b) HCA of GLF (batch 15 is the standard); (c) PCA of GLS; (d) HCA of GLS (batch 10 is the standard)

very low percentage only in the standard GLF samples $(0.006 \mathrm{mg} / \mathrm{g})$, so it can also be excluded as a chemical marker. Based on the above findings, the 11 Ganoderma acids would be the appropriate chemical markers for quality control for GLS and GLF samples. If only the Ganoderma acids are included as chemical markers, the 
alkalized buffer with $\mathrm{pH}$ around 8 can be tried to increase the extraction rate because the solubility of the acids is dramatically higher in basic conditions based on our Scifinder data search. This needs to be further investigated to improve the extraction rate.

Some compounds are similar in their structures (see Fig. 1), making it very difficult to separate them with a good resolution in HPLC chromatograms. For example, some of the compounds only have very little difference in their structures, such as a double bond versus single bond (GAC vs GAC2; GAD vs GAND), or carbonyl group vs hydroxyl group. We have tried to optimize the gradient elution of the mobile phases. It appeared not possible to separate them only by changing the composition of the mobile phases. We then tried to use a column with a smaller particle size since the smaller particle size of the column can lead to higher theoretical plate counts. The results showed all the peaks could be reasonably resolved. In order to achieve better separation, an amino or ion exchange column can be tried in the future. Because the content of most components is low, and a lot of interface from other unknown gradients, an HPLC-MS/MS method can be developed to verify the content of these compounds.

This study found that the fruiting body contained much more small chemical components than the spore samples. Although the quantification of chemical markers is a well-accepted quality control method for herbal medicines, it may be an appropriate approach for GL spore products, as the small chemicals are presented the minor portions compared to GL fruiting body products. Such information could be useful for establishing the quality standards for the commercial product made from GL spores.

The quality of the GL product (GLS or GLF) appeared to be not consistent for those collected in different years. Especially for the samples collected before and after 2017. As indicated by the similarity values and PCA, the samples collected before 2017 are closed to each other, but quite different from those after 2017. To further figure out the possible reason, a detailed analysis of the condition of planting, growing, and processing is needed, which may provide an insight to improve the quality consistency of GL products.

\section{CONCLUSIONS}

A validated HPLC-DAD assay method has been successfully developed for the quantification of the 13 active components in herbal products of GLS, GLF, and GLSF mixtures. The 11 Ganoderma acids appeared to be good chemical markers for quality control of GLS and GLF products. The fruiting body samples contained much more abundant of these components than spore samples. The HPLC-DAD fingerprints showed the quality of the GLS and GLF samples are not consistent. The year of the harvesting could be a significant factor contributing to the variations.

\section{REFERENCES}

1. Zhou, L. W.; Cao, Y.; Wu, S.-H.; Vlasák, J.; Li, D.-W.; Li, M.-J.; Dai, Y.-C. Global diversity of the Ganoderma lucidum complex (Ganodermataceae, Polyporales) inferred from morphology and multilocus phylogeny. Phytochemistry 2015, 114, 7-15. https://doi. org/10.1016/j.phytochem.2014.09.023.

2. Lindequist, U.; Jülich, W.-D.; Witt, S. Ganoderma pfeifferi - a European relative of Ganoderma lucidum. Phytochemistry 2015, 114,102-8. https://doi.org/10.1016/j.phytochem.2015.02.018.

3. Cizmarikova, M. The efficacy and toxicity of using the lingzhi or Reishi medicinal mushroom, Ganoderma lucidum (agaricomycetes), and its products in chemotherapy (review). Int. J. Med. Mushrooms 2017, 19(10), 861-77. https://doi.org/10.1615/IntJMedMushrooms. 2017024537.

4. Guggenheim, A. G.; Wright, K. M.; Zwickey, H. L. Immune modulation from five major mushrooms: application to integrative oncology. Integr. Med. (Encinitas, Calif.) 2014, 13(1), 32-44.

5. Paterson, R. R. M. Ganoderma - a therapeutic fungal biofactory. Phytochemistry 2006, 67(18), 1985-2001. https://doi.org/10.1016/j. phytochem.2006.07.004.

6. Soccol, C. R.; Bissoqui, L. Y.; Rodrigues, C.; Rubel, R.; Sella, S. R.; Leifa, F.; de Souza Vandenberghe, L. P.; Soccol, V. T. Pharmacological properties of biocompounds from spores of the lingzhi or Reishi medicinal mushroom ganoderma lucidum (agaricomycetes): a review. Int. J. Med. Mushrooms 2016, 18(9), 757-67. https://doi. org/10.1615/IntJMedMushrooms.v18.i9.10.

7. Cheng, S.; Sliva, D. Ganoderma lucidum for cancer treatment: we are close but still not there. Integr. Cancer Ther. 2015, 14(3), 249-57. https://doi.org/10.1177/1534735414568721.

8. Liang, C.; Tian, D.; Liu, Y.; Li, H.; Zhu, J.; Li, M.; Xin, M.; Xia, J. Review of the molecular mechanisms of Ganoderma lucidum triterpenoids: ganoderic acids A, C2, D, F, DM, X and Y. Eur. J. Med. Chem. 2019, 174, 130-41. https://doi.org/10.1016/j.ejmech.2019.04. 039.

9. Necyk, C.; Zubach-Cassano, L. Natural health products and diabetes: a practical review. Can. J. Diabetes 2017, 41(6), 642-7. https://doi.org/10.1016/j.jcjd.2017.06.014.

10. Liu, W.; Zhang, J.; Han, W.; Liu, Y.; Feng, J.; Tang, C.; Feng, N.; Tang, Q. One single standard substance for the simultaneous determination of 17 triterpenes in Ganoderma lingzhi and its related species using high-performance liquid chromatography. J. Chromatogr. B 2017, 1068-1069, 49-55. https://doi.org/10.1016/j. jchromb.2017.10.010.

11. Yan, Z.; Xia, B.; Qiu, M. H.; Li Sheng, D.; Xu, H. X. Fast analysis of triterpenoids in Ganoderma lucidum spores by ultra-performance liquid chromatography coupled with triple quadrupole mass spectrometry. Biomed. Chromatogr. : BMC 2013, 27(11), 1560-7. https:// doi.org/10.1002/bmc.2960.

12. Zhao, J.; Zhang, X. Q.; Li, S. P.; Yang, F. Q.; Wang, Y. T.; Ye, W. C. Quality evaluation of Ganoderma through simultaneous determination of nine triterpenes and sterols using pressurized liquid extraction and high performance liquid chromatography. J. Sep. Sci. 2006, 29(17), 2609-15. https://doi.org/10.1002/jssc.200600178.

13. Liu, Y.; Liu, Y.; Qiu, F.; Di, X. Sensitive and selective liquid chromatography-tandem mass spectrometry method for the determination of five ganoderic acids in Ganoderma lucidum and its 
related species. J. Pharm. Biomed. Anal. 2011, 54(4), 717-21. https://doi.org/10.1016/j.jpba.2010.11.002.

14. Zhang, H.; Jiang, H.; Zhang, X.; Tong, S.; Yan, J. Development of global chemical profiling for quality assessment of ganoderma species by ChemPattern software. J. Anal. Methods Chem. 2018, 1675721. https://doi.org/10.1155/2018/1675721.

15. Di, X.; Chan, K. K. C.; Leung, H. W.; Huie, C. W. Fingerprint profiling of acid hydrolyzates of polysaccharides extracted from the fruiting bodies and spores of Lingzhi by high-performance thinlayer chromatography. J. Chromatogr. 2003, 1018(1), 85-95. https://doi.org/10.1016/j.chroma.2003.07.015.

16. Gao, J.; Sato, N.; Hattori, M.; Ma, C.-M. The simultaneous quantification of Ganoderma acids and alcohols using ultra high-performance liquid chromatography-mass spectrometry in dynamic selected reaction monitoring mode. J. Pharm. Biomed. Anal. 2013, 74, 246-9. https://doi.org/10.1016/j.jpba.2012.11.003.
17. Wu, L.; Liang, W.; Chen, W.; Li, S.; Cui, Y.; Qi, Q.; Zhang, L. Screening and analysis of the marker components in ganoderma lucidum by HPLC and HPLC-MS(n) with the aid of chemometrics. Molecules 2017, 22(4), 584. https://doi.org/10.3390/ molecules22040584.

18. Ahmad, M. F. Ganoderma lucidum: persuasive biologically active constituents and their health endorsement. Biomed. Pharmacother. 2018, 107, 507-19. https://doi.org/10.1016/j.biopha.2018.08.036.

19. Hu, G.; Zhai, M.; Niu, R.; Xu, X.; Liu, Q.; Jia, J. Optimization of culture condition for ganoderic acid production in ganoderma lucidum liquid static culture and design of a suitable bioreactor. Molecules 2018, 23(10), 2563. https://doi.org/10.3390/ molecules 23102563.

20. Weete, J. D.; Abril, M.; Blackwell, M. Phylogenetic distribution of fungal sterols. PLoS One 2010, 5(5), e10899. https://doi.org/10. 1371/journal.pone.0010899. 